\title{
Significance of connective tissue disease features in idiopathic interstitial pneumonia
}

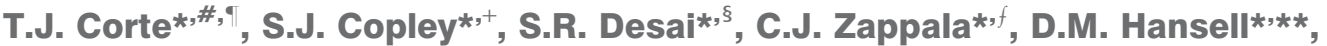

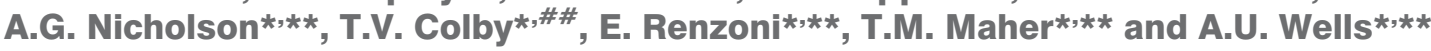

ABSTRACT: In idiopathic interstitial pneumonia (IIP), the significance of connective tissue disease (CTD) features in the absence of a specific CTD diagnosis remains unclear. We studied the clinical and prognostic utility of a diagnosis of undifferentiated CTD (UCTD) in patients with biopsy-proven IIP.

IIP patients undergoing surgical lung biopsy (1979-2005) were studied (nonspecific interstitial pneumonia (NSIP), $n=45$; idiopathic pulmonary fibrosis, $n=56$ ). UCTD was considered present when serum autoantibodies were present and symptoms or signs suggested CTD. The relationship between UCTD and NSIP histology was evaluated. A clinical algorithm that best predicted NSIP histology was constructed using a priori variables. The prognostic utility of UCTD, and of this algorithm, was evaluated.

UCTD was present in $14(31 \%)$ NSIP and seven (13\%) IPF patients. UCTD was not associated with a survival benefit. The algorithm predictive of NSIP (OR 10.4, 95\% $\mathrm{Cl} 3.21-33.67 ; p<0.0001$ ) consisted of the absence of typical high-resolution computed tomography (HRCT) features for IPF and 1) a compatible demographic profile (females aged $<50 \mathrm{yrs}$ ) or 2) Raynaud's phenomenon. In patients with an HRCT scan not typical for IPF, this algorithm predicted improved survival (hazard ratio $0.35,95 \% \mathrm{Cl} 0.14-0.85 ; \mathrm{p}=0.02$ ) independent of IIP severity.

UCTD is associated with NSIP histology. However, the diagnostic and prognostic significance of UCTD in IIP patients remains unclear.

KEYWORDS: Connective tissue disease, idiopathic interstitial pneumonia, idiopathic pulmonary fibrosis, nonspecific interstitial pneumonia, prognosis, undifferentiated connective tissue disease

$\mathbf{N}$ onspecific interstitial pneumonia (NSIP) is the most common histological finding when patients fulfilling American College of Rheumatology (ACR) criteria for specific connective tissue diseases (CTDs) undergo lung biopsy for investigation of fibrotic lung disease [1-3]. However, up to $25 \%$ of patients with features of CTD do not fulfil ACR criteria for specific CTD, and are considered to have undifferentiated CTD (UCTD) [4-8]. UCTD is usually considered present when patients have signs or symptoms suggestive of CTD and positive autoimmune serology for $>12$ months [4, 5, 7, 9-11]. A minority of patients with UCTD develop specific CTD over time $[4-8,12]$.

It is not uncommon for patients with idiopathic interstitial pneumonia (IIP) to have symptoms and serological findings consistent with UCTD [13-15]. A recent expert commentary has highlighted the fact that many IIP patients do not satisfy specific CTD diagnostic criteria, but clearly have significant end-organ disease [16]. The majority of patients with these autoimmune features have NSIP at surgical lung biopsy [17, 18]. Thus, it has been proposed that UCTD manifests in the lung as "idiopathic NSIP" [9]. The distinction of idiopathic NSIP from idiopathic pulmonary fibrosis (IPF) has important prognostic and treatment implications [19-21]. However, a confident diagnosis of NSIP requires surgical lung biopsy. In a recent study using a broad, and somewhat controversial, definition of UCTD, KINDER et al. [9] showed that UCTD is common in patients with IIP, especially in a small subset of 17 patients with biopsy-proven idiopathic NSIP $[9,16]$. On this basis, those authors suggested that a diagnosis of UCTD may be able to distinguish patients with idiopathic NSIP before obtaining surgical lung biopsy [9].

Based upon these a priori observations, we first aimed to determine the relationship between the diagnosis of UCTD and NSIP histology in a large cohort of patients with biopsy-proven idiopathic NSIP and IPF. Secondly, we aimed to explore the clinical utility of a diagnosis of UCTD, with regard to the identification of NSIP at surgical lung biopsy and in the prognostic evaluation of IIP patients.
AFFILIATIONS

${ }^{*}$ Dept of Respiratory Medicine, Royal Brompton Hospital and National Heart and Lung Institute, ${ }^{+}$Dept of Radiology, Hammersmith Hospital, Imperial NHS Trust, ${ }^{\text {s}}$ Dept of Radiology, King's College Hospital Foundation NHS Trust, ${ }^{* *}$ Dept of Respiratory Medicine, Imperial College, London, UK. \#Dept of Respiratory Medicine, University of Sydney,

"Dept of Respiratory Medicine, Royal Prince Alfred Hospital, Sydney, ${ }^{f}$ Dept of Respiratory Medicine, Royal Brisbane Hospital, Brisbane, Australia.

\#\# Dept of Histopathology, Mayo Clinic, Scottsdale, AZ, USA.

CORRESPONDENCE

A.U. Wells

Interstitial Lung Disease Unit

Royal Brompton Hospital and NHLI Imperial College

Emmanuel Kaye Building

1B Manresa Road

London

SW3 6LP

UK

E-mail: athol.wells@rbht.nhs.uk

Received:

Nov 112010

Accepted after revision:

July 212011

First published online:

Sept 152011

European Respiratory Journal Print ISSN 0903-1936 Online ISSN 1399-3003 


\section{METHODS}

\section{Study subjects}

Subjects were identified from a hospital database $(n=232)$ who met the histological criteria at surgical biopsy and the clinical criteria for idiopathic interstitial pneumonia: IPF $(n=138)$ or idiopathic fibrotic NSIP ( $\mathrm{n}=94)$ between January 1, 1978 and June 30, 2005. 152 patients were identified (IPF, $\mathrm{n}=91$; NSIP, $\mathrm{n}=61$ ) whose clinical notes were available (not destroyed). 20 patients had incomplete clinical and laboratory data, and were excluded from the analysis. We studied the 101 patients (IPF, $n=56$; NSIP, $\mathrm{n}=45$ ) with concurrent high-resolution computed tomography (HRCT).

Data were collected retrospectively from medical records. IPF patients met American Thoracic Society (ATS)/European Respiratory Society (ERS) diagnostic criteria [22]. Clinical criteria for NSIP comprised: 1) bilateral, predominantly basal or widespread crackles; 2) radiographic evidence of predominantly basal, bilateral lung infiltrates with ground-glass or reticular opacities on HRCT; 3) a restrictive functional defect or isolated reduction in diffusing capacity; 4) absence of features suggestive of hypersensitivity pneumonia or organising pneumonia; and 5) no other known cause of pulmonary fibrosis or associated disease. Clinical diagnosis was confirmed by multidisciplinary consensus. No patients met ACR criteria for a specific CTD. The treatment regimen included: 1) combination immunosuppressive treatment including low-dose prednisolone (10 mg); or 2) high-dose prednisolone, reducing to a maintenance dose (mean $10 \mathrm{mg}$ ).

It is our routine practice to question patients for symptoms of CTD, including: Raynaud's phenomenon, arthralgia, myalgia, morning stiffness, Sicca symptoms, gastro-oesophageal reflux or dysphagia, and skin changes. Other symptoms were noted to be present if documented as such in the medical records and assumed absent if not recorded.

Patients were followed up until death, to lung transplantation or to June 1, 2008, with follow-up complete in 93 out of 101 cases. Vital status was determined at June 1, 2008 from hospital databases and confirmed with local physicians. 52 (51\%) deaths occurred during follow-up (median 44 months, range 2-258 months). No patients underwent lung transplantation during the follow-up period. Local ethical committee approval was in place for retrospective studies.

\section{Investigations}

\section{Histopathology}

All lung biopsy specimens were independently reviewed by two histopathologists blinded to the clinical data $(\kappa=0.53)$, with diagnostic divergences resolved by consensus. Histopathological diagnosis was made according to the ATS/ERS international consensus classification of IIPs [23, 24].

\section{HRCT}

All patients underwent concurrent HRCT. HRCT was reviewed by two specialist respiratory radiologists. HRCT diagnosis was considered "typical of IPF", "indeterminate" or "compatible with NSIP". Consensus scores were generated for the extent of disease, and the proportions of ground-glass opacity, fine reticular changes, and micro- and macrocystic changes at each of five levels, and the total extent of each feature was calculated.

\section{Pulmonary function testing}

Pulmonary function testing was performed in all patients (Jaeger Masterscreen 240; Cardinal Health UK Ltd, Basingstoke, UK) and predicted values were calculated according to ATS/ERS guidelines [25-28]. Lung volumes, spirometric volumes and diffusing capacity of the lung for carbon monoxide were measured. The composite physiologic index (CPI) was calculated as previously described [29].

\section{Laboratory testing}

All patients had autoimmune serology, C-reactive protein (CRP) and erythrocyte sedimentation rate (ESR) measurements performed. Antinuclear antibody (ANA) testing was performed using indirect immunofluorescence, and considered positive when $\geqslant 3+$. Extractable nuclear antigen (ENA) and doublestranded DNA analyses were performed using automated ELISA. Specific antibody characterisation was performed in most patients when ENA testing was positive, including anti-Ro (SS-A), anti-La (SS-B), anti-Scl-70, anti-centromere, anti-ribonucleoprotein (RNP) and Jo-1 antibodies. Rheumatoid factor (RF) was analysed using partial agglutination and considered positive when greater than $1 / 160$.

\section{Diagnosis of UCTD}

Patients were considered to have a diagnosis of UCTD (table 1) if they had one of the specific autoantibodies (SS-A, SS-B, anti-Scl70, anti-centromere, anti-RNP or Jo-1), or a positive ENA or ANA titre, as well as symptoms or signs of CTD (including Raynaud's phenomenon, Sicca symptoms, arthralgia, morning stiffness or proximal muscle weakness) [10]. We also considered whether patients met the broader UCTD definition proposed by KINDER et al. [9] (table 1).

\section{Statistical analysis}

All analyses were performed using STATA statistical software (version 10.0; StataCorp, College Station, TX, USA). Data are presented as mean \pm SD or median (range). Group comparisons were made using unpaired t-tests, Wilcoxon's rank-sum test or the Chi-squared test. The sensitivity and specificity of a diagnosis of UCTD for NSIP histology was determined.

Outcome was evaluated for mortality, from the time of surgical lung biopsy, using Cox regression, with satisfaction of the assumptions of proportional hazards analysis, for a priori covariates: 1) diagnosis of UCTD, as well as satisfying the broader definition of UCTD of KINDER et al. [9]; 2) demographic variables (age, female sex and females aged $<50$ yrs, as the mean age of females was 50 yrs); 3) UCTD features (Raynaud's phenomenon, positive autoimmune serology, and one or more of Raynaud's or positive serology); 4) functional severity (CPI); 5) HRCT typical of IPF; and 6) histological diagnosis of IPF.

Multivariate survival analysis was performed for UCTD diagnosis, adjusting for functional severity (CPI) and histological diagnosis.

We set out to construct a clinical algorithm predictive of a histological diagnosis of NSIP using step-wise multiple logistic regression analysis. A priori covariates included absence of HRCT typical of IPF, a compatible demographic profile (females aged $<50 \mathrm{yrs}$ ) and UCTD features (Raynaud's phenomenon and positive autoimmune serology). The sensitivity and specificity 


\section{TABLE 1 Diagnostic criteria for undifferentiated connective tissue disease (UCTD)}

\section{A. Symptoms associated with CTD}

B. Positive autoimmune serology
At least one of: 1) Raynaud's phenomenon; 2) arthralgias/multiple joint swelling; 3) morning stiffness; 4) dry mouth or dry eyes (Sicca features); 5) proximal muscle weakness
At least one of: 1) Raynaud's phenomenon; 2) arthralgias/multiple joint swelling; 3) photosensitivity; 4) unintentional weight loss; 5) morning stiffness; 6) dry mouth or dry eyes (Sicca features);

7) dysphagia; 8) recurrent unexplained fever; 9) gastro-oesophageal reflux; 10) skin changes (rash); 11) oral ulceration;

12) nonandrogenic alopecia; 13) proximal muscle weakness Positive finding of at least one of:

Positive finding of at least one of: 1) ANA (high titre); 2) RF (high titre); 3) positive ENA; 4) anti-Scl70 antibody; 5) anti-RNP antibody; 6) anticentromere antibody; 7) SS-A or SS-B; 8) Jo-1 antibody
1) ANA; 2) RF; 3) anti-Scl70 antibody; 4) SS-A or SS-B; 5) Jo-1 antibody; 6) ESR (>2 times normal), CRP

CTD: connective tissue disease; ANA: antinuclear antibody; RF: rheumatoid factor; ENA: extractable nuclear antigen; RNP: ribonucleoprotein; SS-A: anti-Ro; SS-B: antiLa; ESR: erythrocyte sedimentation rate; CRP: C-reactive protein. ${ }^{*}$ : a diagnosis of UCTD requires having one or more of the symptoms outlined in A, and one or more of the laboratory findings listed in $\mathrm{B} ; "$ : criteria proposed by KINDER et al. [9].

of this algorithm for NSIP histology was determined. The prognostic value of this algorithm was determined using multivariate Cox survival analysis.

\section{RESULTS}

\section{Patient characteristics}

The mean age of the cohort was 54 yrs and 38 (38\%) out of 101 were female. As shown in table 2, patients with NSIP were younger $(p=0.003)$ and more likely to be female $(p<0.0001)$. Pulmonary function tests did not differ between IPF and NSIP. The most common systemic symptom was gastro-oesophageal reflux (39\%), followed by arthralgia (24\%), Sicca symptoms $(19 \%)$, Raynaud's phenomenon (13\%) and lethargy (13\%). Raynaud's phenomenon $(\mathrm{p}<0.0001)$ was more common in NSIP but no other systemic symptom was associated with histological subtype. NSIP patients were more likely to present with wheeze $(\mathrm{p}<0.0001)$.

The most common laboratory findings were elevation of CRP (39\%), elevated ESR (19\%), positive ANA (15\%), positive ENA $(19 \%)$ and positive RF (9\%) (table 3). Patients with NSIP were more likely to have any positive autoimmune serology $(\mathrm{p}=0.007)$ than IPF patients.

UCTD was present in 21 (21\%) out of 101 patients and was more frequent in NSIP (14 out of 45,31\%) than in IPF (seven out of 56, $13 \%)(p=0.02)$. The diagnosis of UCTD was associated with a three-fold increase in the likelihood of NSIP (OR 3.16, 95\% CI $1.14-8.70 ; p=0.03$ ), and had a sensitivity of $31 \%$ and a specificity of $88 \%$ for NSIP histology (table 4 ).

We also considered the prevalence of the broader definition of UCTD of KINDER et al. [9], and found that these criteria were satisfied in a higher proportion of patients overall (52 out of 101, $52 \%$ ) and were also more frequent in NSIP (32 out of $45,71 \%$ ) than IPF (20 out of $56,36 \%$ ). There was a similar association between this broader definition of UCTD and NSIP (OR 4.43, 95\% CI $1.90-10.32 ; p=0.001$ ), with a sensitivity of $71 \%$ and a specificity of $64 \%$ for NSIP histology.

\section{Survival analysis}

As shown in table 5, the diagnosis of UCTD was not associated with improved survival (hazard ratio (HR) 1.07, 95\% CI 0.54-2.10; $\mathrm{p}=0.85$ ) (fig. 1). This was also true for the broader definition of
UCTD of KINDER et al. [9]. No single CTD symptom, sign or laboratory test predicted survival. Functional impairment (CPI) (HR 1.04, 95\% CI 1.02-1.06; $\mathrm{p}<0.0001)$ and usual interstitial pneumonia (UIP) histology (HR 3.71, 95\% CI 2.00-6.89; p <0.0001) were associated with increased mortality, whereas female sex was associated with lower mortality (HR 0.48, 95\% CI 0.25-0.90; $\mathrm{p}=0.02)$ and age was not predictive of survival.

\section{HRCT appearance}

As shown in table 6, IPF on surgical biopsy was associated with increased fine reticular $(p=0.0004)$, microcystic $(p=0.0001)$ and macrocystic $(p=0.03)$ changes on HRCT, whereas NSIP histology was linked to increased ground-glass changes $(p=0.0008)$. By contrast, HRCT findings did not differ between patients with and without UCTD, either in the whole cohort or within histological subgroups.

\section{A clinical algorithm predictive of a histological diagnosis of NSIP}

Patients with HRCT appearances typical of IPF $(n=23,23 \%)$ had a low likelihood of NSIP (OR 0.19, 95\% CI 0.06-0.61; $\mathrm{p}=0.005$ ).

When HRCT appearance was not typical of IPF, the histological diagnosis was UIP in 37 (47\%) out of 78 and NSIP in 41 (53\%) out of 78 patients. In patients with HRCT not typical of IPF, NSIP was associated with female sex (OR 4.4, 95\% CI 1.66-11.64; $\mathrm{p}=0.003$ ) and younger age (OR 0.94, 95\% CI 0.89-0.99; $\mathrm{p}=0.02)$. Females aged $<50$ yrs $(n=16,21 \%)$ were likely to have NSIP (OR 5.26, 95\% CI 1.36-20.32; $\mathrm{p}=0.02$ ). However, specific clinical or laboratory features of UCTD were not associated with NSIP within this demographic group, which was excluded from subsequent analysis. In the remaining 62 patients, the presence of Raynaud's phenomenon (OR 10.67, 95\% CI 1.22-93.08; $p=0.03$ ) was more strongly associated than positive autoimmune serology (OR 2.89, 95\% CI 0.95-8.85; $\mathrm{p}=0.06$ ) or one of the above (OR 3.75, 95\% CI 1.27-11.11; $\mathrm{p}=0.02)$ with NSIP.

Based on these findings, the optimal algorithm identifying a high likelihood of NSIP consisted of the absence of HRCT features typical of IPF and the presence of either: 1) a compatible demographic profile (females aged $<50 \mathrm{yrs}$ ); or 2) Raynaud's phenomenon (fig. 2). These criteria, which were satisfied in 24 (24\%) out of 


\begin{tabular}{|c|c|c|c|c|}
\hline \multirow{2}{*}{ TABLE 2} & \multicolumn{4}{|c|}{$\begin{array}{l}\text { Demographic and clinical characteristics of } \\
\text { patients with biopsy-proven nonspecific } \\
\text { interstitial pneumonia (NSIP) compared with } \\
\text { patients with idiopathic pulmonary fibrosis (IPF) }\end{array}$} \\
\hline & & IPF & NSIP & p-value ${ }^{\#}$ \\
\hline Subjects & & 56 & 45 & \\
\hline \multicolumn{5}{|c|}{ Clinical features } \\
\hline Age at first & resentation yrs & $56 \pm 9$ & $50 \pm 10$ & 0.003 \\
\hline Males & & $44(79)$ & $19(42)$ & $<0.0001$ \\
\hline Never-smol & & $19(34)$ & $24(55)$ & 0.05 \\
\hline $\begin{array}{c}\text { Duration of } \\
\text { months }\end{array}$ & espiratory symptoms & $15(0-120)$ & $20(0-156)$ & 0.60 \\
\hline Dyspnoea & & $55(98)$ & $43(95)$ & 0.43 \\
\hline Cough & & $46(82)$ & $36(80)$ & 0.784 \\
\hline Wheeze & & $21(38)$ & $23(51)$ & $<0.0001$ \\
\hline Clubbing & & $28(50)$ & $16(36)$ & 0.17 \\
\hline Bibasal ins & ratory crackles & $51(91)$ & $41(91)$ & 0.70 \\
\hline FVC \% pre & & $69 \pm 20$ & $69 \pm 22$ & 0.95 \\
\hline DL,CO \% pr & & $42 \pm 17$ & $44 \pm 15$ & 0.60 \\
\hline $\mathrm{CPI}$ & & $52 \pm 14$ & $49 \pm 14$ & 0.38 \\
\hline BAL lymph & ytes \% & $9.6 \pm 6.6$ & $12.7 \pm 10.4$ & 0.15 \\
\hline \multicolumn{5}{|c|}{ UCTD features } \\
\hline Arthralgia & & $10(29)$ & $14(31)$ & 0.15 \\
\hline Myalgia & & $5(9)$ & $1(2)$ & 0.15 \\
\hline Lethargy & & $6(11)$ & 7 (16) & 0.49 \\
\hline Gastro-oes & ohageal reflux & $21(38)$ & $18(40)$ & 0.85 \\
\hline Raynaud's & henomenon & 124) & $12(27)$ & $<0.0001$ \\
\hline Dysphagia & & $0(0)$ & $1(2)$ & 0.27 \\
\hline Sicca symp & oms & 8 (19) & $11(20)$ & 0.21 \\
\hline Recurrent $f$ & & $3(5)$ & $4(9)$ & 0.48 \\
\hline Skin chang & & 7 (13) & $5(11)$ & 0.81 \\
\hline Morning sti & ness & $3(6)$ & $6(13)$ & 0.18 \\
\hline Unintention & weight loss & $8(14)$ & $5(11)$ & 0.62 \\
\hline Proximal $\mathrm{m}$ & scle weakness & $1(2)$ & $3(7)$ & 0.24 \\
\hline Oral ulcera & & $1(2)$ & $1(2)$ & 0.89 \\
\hline Photosensi & & $0(0)$ & $0(0)$ & \\
\hline Alopecia, n & nandrogenic & $0(0)$ & $2(4)$ & 0.11 \\
\hline
\end{tabular}

Data are presented as $n$, mean \pm SD, $n$ (\%) or median (range), unless otherwise stated. FVC: forced vital capacity; \% pred: \% predicted; DL,CO: diffusing capacity of the lung for carbon monoxide; $\mathrm{CPI}$ : composite physiologic index; BAL: bronchoalveolar lavage; UCTD: undifferentiated connective tissue disease. ${ }^{\#}$ : unpaired t-test, Chi-squared test or Fisher's exact test.

101 patients, were strongly associated with NSIP (OR 10.40, 95\% CI 3.21-33.67; $\mathrm{p}<0.0001$ ), with a low sensitivity ( 20 out of $44,44 \%$ ) but a very high specificity (55 out of 56, 93\%) (table 4 ).

In order to evaluate the prognostic value of this algorithm, outcome was first evaluated against HRCT. Patients with appearances typical of IPF had a higher mortality (HR 2.82, 95\% CI 1.54-5.15; $\mathrm{p}=0.001$ ), after adjustment for CPI levels, which were independently positively associated with mortality. In the remaining patients, with HRCT appearances not typical of IPF, mortality was lower in patients satisfying algorithm inclusion criteria (females aged $<50$ yrs or the presence of Raynaud's phenomenon) (HR 0.35, 95\% CI 0.14-0.85; p=0.02) (table 7) following adjustment for disease severity (CPI). Similarly,
TABLE 3 Laboratory data for patients with biopsy-proven nonspecific interstitial pneumonia (NSIP) compared to patients with idiopathic pulmonary fibrosis (IPF)

\begin{tabular}{lccc} 
Characteristics & IPF & NSIP & p-value ${ }^{\#}$ \\
\hline $\begin{array}{l}\text { Subjects } \\
\text { Autoimmune serology }\end{array}$ & 56 & 45 & \\
$\quad$ ANA positive & $6(11)$ & $9(20)$ & 0.21 \\
ENA positive & $2(4)$ & $7(16)$ & 0.04 \\
dsDNA positive & $1(2)$ & $3(7)$ & 0.23 \\
SS-A or SS-B antibody positive & $0(0)$ & $1(0)$ & \\
Anti-Scl70 antibody positive & $0(0)$ & $2(4)$ & 0.11 \\
Anti-Jo1 antibody positive & $0(0)$ & $2(4)$ & 0.27 \\
RF positive & $3(5)$ & $6(13)$ & 0.17 \\
ANCA positive & 0 & $2(6)$ & 0.80 \\
Any autoimmune serology positive & $12(21)$ & $21(47)$ & 0.007 \\
CRP $\mathbf{~ m g} \cdot \mathbf{L}^{-1}$ & $36 \pm 9$ & $14 \pm 14$ & 0.01 \\
ESR $\mathbf{~ m m} \cdot \mathbf{h}^{-1}$ & $21 \pm 21$ & $16 \pm 18$ & 0.23 \\
\hline
\end{tabular}

Data are presented as $n, n(\%)$ or mean $\pm S D$, unless otherwise stated. ANA antinuclear antibody; ENA: extractable nuclear antigen; dsDNA: doublestranded DNA; SS-A: anti-Ro; SS-B: anti-La; RF: rheumatoid factor; ANCA: anti-neutrophilic cytoplasmic antibody; CRP: C-reactive protein; ESR: erythrocyte sedimentation rate. ${ }^{*}$ : Wilcoxon's rank-sum test or Chi-squared test.

mortality was lower if a diagnosis of UCTD was substituted for the presence of Raynaud's phenomenon (HR 0.38, 95\% CI 0.160.91; $\mathrm{p}=0.03)$. However, the histological diagnosis of IPF remained the strongest prognostic marker in this patient group (HR 4.18, 95\% CI 1.93-9.00; $\mathrm{p}<0.0001$ ).

\section{DISCUSSION}

We report that CTD features are not uncommon in so-called IIP, with $21 \%$ of NSIP and $13 \%$ of IPF patients fulfilling Mosca's diagnostic criteria for UCTD [10]. When the less specific diagnostic criteria for UCTD of KINDER et al. [9] were applied, $71 \%$ of NSIP and $36 \%$ of IPF patients were found to have UCTD. It is not certain whether the classification of patients with CTD features who do not fulfil specific CTD criteria as having a diagnosis of UCTD has either clinical or prognostic significance.

TABLE 4 Sensitivity and specificity of undifferentiated connective tissue disease (UCTD) diagnostic criteria for nonspecific interstitial pneumonia (NSIP) histology

\begin{tabular}{|c|c|c|c|c|}
\hline Diagnostic criteria for UCTD & Sensitivity & Specificity & PPV & NPV \\
\hline UCTD diagnostic criteria & 31 & 88 & 67 & 61 \\
\hline Broader definition of UCTD\# & 71 & 64 & 62 & 59 \\
\hline $\begin{array}{l}\text { UCTD algorithm predictive for } \\
\text { NSIP }\end{array}$ & 44 & 93 & 83 & 68 \\
\hline
\end{tabular}

Data are presented as \%. PPV: positive predictive value; NPV: negative predictive value. ${ }^{\#}$ : criteria proposed by KINDER et al. [9]. 


\begin{tabular}{|c|c|c|}
\hline Characteristics & $\mathrm{HR}(95 \% \mathrm{Cl})$ & $p$-value \\
\hline Diagnosis of UCTD\# & $1.07(0.54-2.10)$ & 0.85 \\
\hline \multicolumn{3}{|l|}{ Clinical features } \\
\hline Age & $1.01(0.99-1.05)$ & 0.24 \\
\hline Female & $0.48(0.25-0.90)$ & 0.02 \\
\hline Female $<50$ yrs & $0.35(0.12-0.96)$ & 0.04 \\
\hline Raynaud's phenomenon & $0.65(0.26-1.64)$ & 0.36 \\
\hline Any one autoimmune serology positive & $0.68(0.37-1.26)$ & 0.22 \\
\hline $\mathrm{CPI}$ & $1.04(1.02-1.06)$ & $<0.0001^{\circ}$ \\
\hline Histological diagnosis of IPF & $3.71(2.00-6.89)$ & $<0.0001^{\bullet}$ \\
\hline \multicolumn{3}{|c|}{$\begin{array}{l}\text { HR: hazard ratio; UCTD: undifferentiated connective tissue disease; CPI: } \\
\text { composite physiologic index; IPF: interstitial pulmonary fibrosis. "\#: pre- } \\
\text { specified criteria outlined in table } 1 ; " \text { : remained significant following adjust- } \\
\text { ment for age and sex. }\end{array}$} \\
\hline
\end{tabular}

Although we showed an association between UCTD and NSIP, this link was not as strong as in previous observations [9]. The diagnosis of UCTD provided no additional prognostic information over the histological IIP diagnosis. We constructed a clinical algorithm, which was specific for NSIP histology. In the absence of histological information or HRCT findings typical of IPF, patients fulfilling this clinical algorithm or the diagnostic criteria for UCTD had improved survival, following adjustment for CPI. Thus, the diagnosis of UCTD may have prognostic value in IIP patients not undergoing diagnostic surgical biopsy, particularly when HRCT features are not typical of IPF.

We were unable to reproduce earlier findings that suggested that UCTD might almost invariably be associated with idiopathic NSIP. Although UCTD, as defined by KINDER et al. [9], was present in $\sim 70 \%$ of NSIP patients, the highly nonspecific nature of the KINDER et al. [9] criteria was underlined by their satisfaction in a third of patients with IPF. Indeed, in the current study, $52 \%$ of patients fulfilled the broader definition of UCTD of KINDER et al. [9], which was only moderately specific $(64 \%)$ but relatively sensitive $(71 \%)$ for NSIP, compared with $21 \%$ of patients who satisfied more standard UCTD criteria, which were relatively specific $(88 \%)$ but not sensitive (31\%) for NSIP histology. Although UCTD diagnostic criteria specific for NSIP histology are empirically desirable, particularly when surgical biopsy is not possible, standard diagnostic criteria were not sensitive for NSIP. In contrast, the less specific criteria of KINDER et al. [9] were more sensitive for NSIP histology, potentially resulting in misclassification of IPF patients as NSIP. However, the clinical or prognostic impact of such misclassification is not certain. The validity of the diagnostic criteria of KINDER et al. [9] has been questioned by rheumatologists and our findings cast further doubt on their value [16]. Above all, the suggestion that these criteria might be used as a substitute for a diagnostic biopsy in distinguishing between IPF and NSIP [9] cannot be countenanced, judging from findings in the present study, and nor did the use of the criteria of KINDER et al. [9] add value to prognostic evaluation, once the histological pattern had been taken into account. In summary, our findings do not support the future use of the criteria of KINDER et al. [9] for the identification of UCTD in clinical practice.

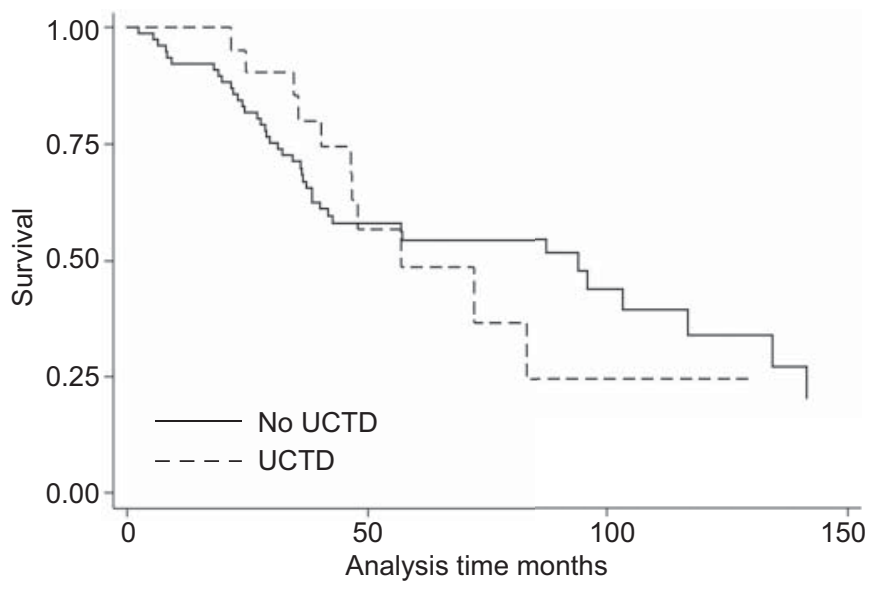

FIGURE 1. Kaplan-Meier survival curve for patients with and without a diagnosis of undifferentiated connective tissue disease (UCTD).

In this regard, a major problem facing clinicians is the lack of a standardised definition for UCTD, now appreciated to be a separate clinical entity $[4,5,7,10,11]$. Since LERoY et al. [30] first proposed the concept of UCTD, there have been more than 10 published diagnostic criteria for UCTD [6, 7, 10, 31-39]. Most definitions are based upon some variation of signs and symptoms suggestive of CTD, positive autoimmune serology and disease duration $>1$ yr $[4,5,7,10,11]$. In some studies, broader definitions of UCTD have been used [9], but, as discussed above, there are major problems with this approach, as UCTD features are nonspecific, and symptoms such as weight loss and proximal muscle weakness are also common in advanced lung disease [40, 41]. In our study, $13 \%$ had unintentional weight loss and $4 \%$ had proximal muscle weakness.

A recent expert commentary on this issue highlights the dilemma of patients with so called IIP who have features suggestive of CTD. Many IIP patients do not fulfil CTD diagnostic criteria, but clearly have significant disease [16]. In that commentary, the authors suggested that these patients in whom there is "a rheumatologic flavor as supported by specific autoantibodies or histopathologic features", but who do not meet criteria for a defined CTD, may be considered to have "lung-dominant CTD" [16]. In our study, we addressed this problem by studying the prevalence and utility of a diagnosis of UCTD in a cohort of patients considered to have IIP. We defined UCTD as the presence of autoantibodies and symptoms/signs suggestive of CTD. The major limitation of our retrospective study was the large time period over which the biopsies were performed. During this time period, laboratory tests were refined, moving to routine testing of specific autoantibodies when ENA tested positive. Despite this limitation, we found that the presence of UCTD was associated with NSIP histology, but portended no survival advantage over patients without UCTD.

In the current study, we show a three-fold increase in NSIP histology among patients with UCTD. This is consistent with several studies that show a clear link between NSIP histology and CTD $[9,42,43]$. Two recent studies have demonstrated that $10 \%$ and $52 \%$ of their patients who were considered to have idiopathic NSIP developed CTD during the follow-up period [43, 44]. In another study, a close association between NSIP histology and 


\begin{tabular}{|c|c|c|c|c|c|c|}
\hline \multirow[t]{3}{*}{ TABLE 6} & atures acc & to biopsy dias & and the & רee of undiffer & ed connective & disease \\
\hline & \multicolumn{3}{|c|}{ Histological diagnosis } & \multicolumn{3}{|c|}{ UCTD } \\
\hline & IPF & NSIP & p-value ${ }^{\#}$ & UCTD positive & UCTD negative & p-value ${ }^{\#}$ \\
\hline Subjects & 56 & 45 & & 21 & 80 & \\
\hline Fine reticular change $\%$ & $17.3(0-57.6)$ & $10.3(0-41.1)$ & 0.0004 & $13.0(0-57.6)$ & $14.7(0-57.2)$ & 0.22 \\
\hline Microcystic change \% & $5.65(0-36.9)$ & $1.8(0-20.6)$ & 0.0001 & $3.0(0-29.9)$ & $4.4(0-36.9)$ & 0.59 \\
\hline Macrocystic change \% & $0(0-24.3)$ & $0(0-10.3)$ & 0.03 & $0(0-24.3)$ & $0(0-23)$ & 0.76 \\
\hline
\end{tabular}

UCTD was demonstrated: NSIP was the histological diagnosis in 15 out of 17 patients with UCTD (OR 50; $p=0.0001$ ), leading the authors to surmise that the application of diagnostic criteria for UCTD may identify NSIP before obtaining surgical lung biopsy [9]. The necessity of surgical lung biopsy for the diagnosis of NSIP has been debated [45]. However, in our study, we found that having a diagnosis of UCTD was not sensitive (31\%) but was relatively specific (88\%) for NSIP histology. Our results do not support the use of more specific UCTD diagnostic criteria alone to identify patients with NSIP, but indicate that the diagnosis of NSIP continues to require surgical lung biopsy [46]. However, in the subset of patients in whom surgical biopsy is not practicable, the UCTD diagnostic criteria may be a useful predictor of NSIP histology. This needs to be confirmed in further prospective studies.

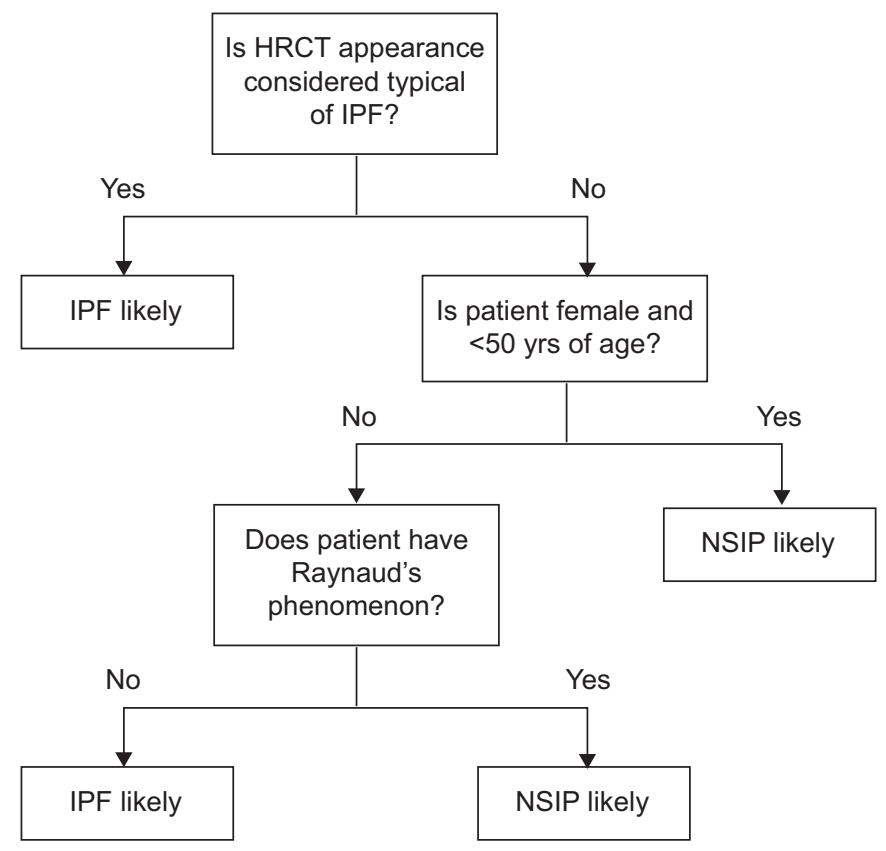

FIGURE 2. Flow diagram for the clinical algorithm predictive of a histological diagnosis of nonspecific interstitial pneumonia (NSIP). HRCT: high-resolution computed tomography; IPF: idiopathic pulmonary fibrosis.
We developed a noninvasive algorithm for the presence of NSIP by testing a priori associations between NSIP and both demographic factors and the presence of UCTD [1-3, 17, 46-48]. The optimal algorithm consisted of the absence of HRCT features typical of IPF and the presence of either: 1) a compatible demographic profile (females aged $<50 \mathrm{yrs}$ ); or 2) Raynaud's phenomenon. $24(24 \%)$ patients fulfilled the criteria for this algorithm, which was associated with a 10-fold increase in likelihood of NSIP. The striking demographic associations with NSIP observed in the present study mirror previous observations $[17,46-49]$, and lend further support for the inclusion of a typical demographic profile in diagnostic and prognostic algorithms. The age threshold of $50 \mathrm{yrs}$ in females evaluated in our study was based upon the statement by an expert group that age $<50$ yrs is an atypical feature in IPF and the fact that 50 yrs was the mean age for females in this study [23].

In the absence of histological information, HRCT findings typical of IPF are widely viewed as diagnostically conclusive. However, when HRCT features are not typical of IPF, both IPF and NSIP histology are frequent, as seen in the current study. In this subgroup, the presence of either a compatible demographic profile (females aged $<50$ yrs) or Raynaud's phenomenon was highly specific for NSIP histology (93\%) and was associated with improved survival, independent of the severity of the underlying fibrosis. We observed similar relationships with the substitution of a diagnosis of UCTD for the presence of Raynaud's

\begin{tabular}{llc} 
TABLE 7 & \multicolumn{3}{l}{$\begin{array}{l}\text { Multivariate survival analysis if high-resolution } \\
\text { computed tomography was not typical of }\end{array}$} \\
idiopathic pulmonary fibrosis & \\
& HR $\mathbf{( 9 5 \% ~ C l )}$ & p-value \\
\hline Raynaud's phenomenon and/or female $<\mathbf{5 0}$ yrs & $0.35(0.14-0.85)$ & 0.02 \\
CPI & $1.05(1.03-1.08)$ & $<0.0001$ \\
Diagnosis of UCTD" and/or female $<\mathbf{5 0}$ yrs & $0.38(0.16-0.91)$ & 0.03 \\
CPI & $1.05(1.03-1.09)$ & $<0.0001$ \\
\hline
\end{tabular}

HR: hazard ratio; CPI: composite physiologic index; UCTD: undifferentiated connective tissue disease. ${ }^{\#}: \mathrm{n}=78$; ${ }^{\bullet}$ : pre-specified criteria outlined in table 1 . 
phenomenon in the above algorithm. Thus, in the absence of histological information and HRCT features typical of IPF, our results support the application of these clinical and demographic features for diagnostic and prognostic assessment of IIP patients. In further support of this conclusion, recent studies have suggested that a diagnosis of UCTD confers a minor prognostic advantage in IIP $[50,51]$.

Our study has several limitations. Unlike the study by KINDER et al. [9], in which a prospective, systematic collection of CTD features was performed, our study was retrospective, spanning two decades. The retrospective design limited our ability to collect comprehensive clinical and serological details. However, patients excluded because of incomplete data did not differ with regard to age, sex and pulmonary function. Although we routinely specifically question patients for the more common symptoms of CTD, other symptoms were considered present if documented and assumed absent if not recorded. To compensate for this potential bias, we first focused upon symptoms for which we had comprehensive data. Secondly, we recognise that in IIP patients undergoing surgical biopsy (a pre-requisite for a diagnosis of NSIP), IIP tends to be less advanced and the HRCT findings less diagnostically conclusive. Thus, the prognostic value of systemic findings in the proposed noninvasive algorithm needs to be further explored in IIP patients with more severe disease. Finally, we recognise the potential risk of "chance fitting" due to the number of variables assessed. This prompted us to examine a priori thresholds, including a single demographic threshold (females aged $<50 \mathrm{yrs}$ ), and restricting UCTD data to components in which we have confidence (Raynaud's phenomenon and positive autoimmune serology). However, despite testing a priori observations, our findings need further evaluation in a separate patient cohort.

\section{Conclusion}

In IIP, the significance of CTD features (in the absence of a specific CTD diagnosis) is not clear. Nonspecific CTD features may be the first, or primary, manifestation of an underlying and undeclared CTD. We demonstrate that it is not uncommon for patients with IIP to display CTD features in the absence of a specific CTD diagnosis, with $21 \%$ of IIP patients fulfilling accepted diagnostic criteria for UCTD. Less specific diagnostic criteria for UCTD that are associated with high prevalence of "UCTD" are not useful. We show that in IIP, the diagnosis of UCTD is associated with NSIP histology, but is neither sensitive nor specific for NSIP, nor associated with a survival advantage. However, the diagnosis of UCTD may have some diagnostic and prognostic significance in the subset of patients in which surgical biopsy is impracticable and HRCT findings are not diagnostic.

\section{STATEMENT OF INTEREST}

None declared.

\section{REFERENCES}

1 Bouros D, Wells AU, Nicholson AG, et al. Histopathologic subsets of fibrosing alveolitis in patients with systemic sclerosis and their relationship to outcome. Am J Respir Crit Care Med 2002; 165: 1581-1586.

2 Douglas WW, Tazelaar HD, Hartman TE, et al. Polymyositisdermatomyositis-associated interstitial lung disease. Am J Respir Crit Care Med 2001; 164: 1182-1185.
3 Ito I, Nagai S, Kitaichi M, et al. Pulmonary manifestations of primary Sjögren's syndrome: a clinical, radiologic, and pathologic study. Am J Respir Crit Care Med 2005; 171: 632-638.

4 Mosca M, Tani C, Neri C, et al. Undifferentiated connective tissue diseases (UCTD). Autoimmun Rev 2006; 6: 1-4.

5 Bodolay E, Csiki Z, Szekanecz Z, et al. Five-year follow-up of 665 Hungarian patients with undifferentiated connective tissue disease (UCTD). Clin Exp Rheumatol 2003; 21: 313-320.

6 Danieli MG, Fraticelli P, Salvi A, et al. Undifferentiated connective tissue disease: natural history and evolution into definite CTD assessed in 84 patients initially diagnosed as early UCTD. Clin Rheumatol 1998; 17: 195-201.

7 Mosca M, Tavoni A, Neri R, et al. Undifferentiated connective tissue diseases: the clinical and serological profiles of 91 patients followed for at least 1 year. Lupus 1998; 7: 95-100.

8 Clegg DO, Williams HJ, Singer JZ, et al. Early undifferentiated connective tissue disease. II. The frequency of circulating antinuclear antibodies in patients with early rheumatic diseases. J Rheumatol 1991; 18: 1340-1343.

9 Kinder BW, Collard HR, Koth L, et al. Idiopathic nonspecific interstitial pneumonia: lung manifestation of undifferentiated connective tissue disease? Am J Respir Crit Care Med 2007; 176: 691-697.

10 Mosca M, Neri R, Bombardieri S. Undifferentiated connective tissue diseases (UCTD): a review of the literature and a proposal for preliminary classification criteria. Clin Exp Rheumatol 1999; 17: 615-620.

11 Doria A, Mosca M, Gambari PF, et al. Defining unclassifiable connective tissue diseases: incomplete, undifferentiated, or both? J Rheumatol 2005; 32: 213-215.

12 Williams HJ, Alarcon GS, Joks R, et al. Early undifferentiated connective tissue disease (CTD). VI. An inception cohort after 10 years: disease remissions and changes in diagnoses in well established and undifferentiated CTD. J Rheumatol 1999; 26: 816-825.

13 Haslam P, Turner Warwick ME, Lukoszek A. Antinuclear antibody and lymphocyte responses to nuclear antigens in patients with lung disease. Clin Exp Immunol 1975; 20: 379-395.

14 Haslam PL, Thomson B, Mohammed I, et al. Circulating immune complexes in patients with cryptogenic fibrosing alveolitis. Clin Exp Immunol 1979; 37: 381-390.

15 Turner-Warwick M, Haslam P. Antibodies in some chronic fibrosing lung diseases. I. Non organ-specific autoantibodies. Clin Allergy 1971; 1: 83-95.

16 Fischer A, West SG, Swigris JJ, et al. Connective tissue diseaseassociated interstitial lung disease: a call for clarification. Chest 2010; 138: 251-256

17 Nagai S, Handa T, Tabuena R, et al. Nonspecific interstitial pneumonia: a real clinical entity? Clin Chest Med 2004; 25: 705-715.

18 Daniil ZD, Gilchrist FC, Nicholson AG, et al. A histologic pattern of nonspecific interstitial pneumonia is associated with a better prognosis than usual interstitial pneumonia in patients with cryptogenic fibrosing alveolitis. Am J Respir Crit Care Med 1999; 160: 899-905.

19 Nicholson AG, Colby TV, du Bois RM, et al. The prognostic significance of the histologic pattern of interstitial pneumonia in patients presenting with the clinical entity of cryptogenic fibrosing alveolitis. Am J Respir Crit Care Med 2000; 162: 2213-2217.

20 Bjoraker JA, Ryu JH, Edwin MK, et al. Prognostic significance of histopathologic subsets in idiopathic pulmonary fibrosis. Am J Respir Crit Care Med 1998; 157: 199-203.

21 Katzenstein AL, Fiorelli RF. Nonspecific interstitial pneumonia/ fibrosis. Histologic features and clinical significance. Am J Surg Pathol 1994; 18: 136-147.

22 American Thoracic Society/European Respiratory Society. Idiopathic pulmonary fibrosis: diagnosis and treatment. International consensus statement. Am J Respir Crit Care Med 2000; 161: 646-664. 
23 American Thoracic Society, European Respiratory Society. American Thoracic Society/European Respiratory Society International Multidisciplinary Consensus Classification of the Idiopathic Interstitial Pneumonias. Am J Respir Crit Care Med 2002; 165: 277-304.

24 Demedts M, Costabel U. ATS/ERS international multidisciplinary consensus classification of the idiopathic interstitial pneumonias. Eur Respir J 2002; 19: 794-796.

25 Macintyre N, Crapo RO, Viegi G, et al. Standardisation of the single-breath determination of carbon monoxide uptake in the lung. Eur Respir J 2005; 26: 720-735.

26 Miller MR, Hankinson J, Brusasco V, et al. Standardisation of spirometry. Eur Respir J 2005; 26: 319-338.

27 Wanger J, Clausen JL, Coates A, et al. Standardisation of the measurement of lung volumes. Eur Respir J 2005; 26: 511-522.

28 Gibson GJ. Standardised lung function testing. Eur Respir J 1993; 6: 155-157.

29 Wells AU, Desai SR, Rubens MB, et al. Idiopathic pulmonary fibrosis: a composite physiologic index derived from disease extent observed by computed tomography. Am J Respir Crit Care Med 2003; 167: 962-969.

30 LeRoy EC, Maricq HR, Kahaleh MB. Undifferentiated connective tissue syndromes. Arthritis Rheum 1980; 23: 341-343.

31 Greer JM, Panush RS. Incomplete lupus erythematosus. Arch Intern Med 1989; 149: 2473-2476.

32 Ganczarczyk L, Urowitz MB, Gladman DD. "Latent lupus". J Rheumatol 1989; 16: 475-478.

33 Alarcon GS, Williams GV, Singer JZ, et al. Early undifferentiated connective tissue disease. I. Early clinical manifestation in a large cohort of patients with undifferentiated connective tissue diseases compared with cohorts of well established connective tissue disease. J Rheumatol 1991; 18: 1332-1339.

34 Danieli MG, Fraticelli P, Franceschini F, et al. Five-year follow-up of 165 Italian patients with undifferentiated connective tissue diseases. Clin Exp Rheumatol 1999; 17: 585-591.

35 Dijkstra S, Nieuwenhuys EJ, Swaak AJ. The prognosis and outcome of patients referred to an outpatient clinic for rheumatic diseases characterized by the presence of antinuclear antibodies (ANA). Scand J Rheumatol 1999; 28: 33-37.

36 Mosca M, Neri R, Bencivelli W, et al. Undifferentiated connective tissue disease: analysis of 83 patients with a minimum followup of 5 years. J Rheumatol 2002; 29: 2345-2349.

37 Bodolay E, Bettembuk P, Balogh A, et al. Osteoporosis in mixed connective tissue disease. Clin Rheumatol 2003; 22: 213-217.

38 Swaak AJ, van de Brink H, Smeenk RJ, et al. Incomplete lupus erythematosus: results of a multicentre study under the supervision of the EULAR Standing Committee on International Clinical Studies Including Therapeutic Trials (ESCISIT). Rheumatology (Oxford) 2001; 40: 89-94.

39 Cavazzana I, Franceschini F, Belfiore N, et al. Undifferentiated connective tissue disease with antibodies to Ro/SSa: clinical features and follow-up of 148 patients. Clin Exp Rheumatol 2001; 19: 403-409.

40 Flaherty KR, Toews GB, Lynch JP 3rd, et al. Steroids in idiopathic pulmonary fibrosis: a prospective assessment of adverse reactions, response to therapy, and survival. Am J Med 2001; 110: 278-282.

41 du Bois RM, Wells AU. Cryptogenic fibrosing alveolitis/idiopathic pulmonary fibrosis. Eur Respir J 2001; 18: Suppl. 32, 43s-55s.

42 Park SW, Ahn MH, Jang HK, et al. Interleukin-13 and its receptors in idiopathic interstitial pneumonia: clinical implications for lung function. J Korean Med Sci 2009; 24: 614-620.

43 Romagnoli M, Nannini C, Piciucchi S, et al. Idiopathic nonspecific interstitial pneumonia: an interstitial lung disease associated with autoimmune disorders. Eur Respir J 2011; 38: 384-391.

44 Park IN, Jegal Y, Kim DS, et al. Clinical course and lung function change of idiopathic nonspecific interstitial pneumonia. Eur Respir J 2009; 33: 68-76.

45 Romagnoli M, Gurioli C, Casoni G, et al. Surgical lung biopsy in the diagnosis of idiopathic NSIP: do we always need it in the initial approach? Am J Respir Crit Care Med 2009; 179: 1071.

46 Travis WD, Hunninghake G, King TE Jr, et al. Idiopathic nonspecific interstitial pneumonia: report of an American Thoracic Society project. Am J Respir Crit Care Med 2008; 177: 1338-1347.

47 Tafti SF, Mokri B, Mohammadi F, et al. Comparison of clinicoradiologic manifestation of nonspecific interstitial pneumonia and usual interstitial pneumonia/idiopathic pulmonary fibrosis: a report from NRITLD. Ann Thorac Med 2008; 3: 140-145.

48 Latsi PI, du Bois RM, Nicholson AG, et al. Fibrotic idiopathic interstitial pneumonia: the prognostic value of longitudinal functional trends. Am J Respir Crit Care Med 2003; 168: 531-537.

49 Monaghan H, Wells AU, Colby TV, et al. Prognostic implications of histologic patterns in multiple surgical lung biopsies from patients with idiopathic interstitial pneumonias. Chest 2004; 125: 522-526.

50 Suda T, Kono M, Nakamura Y, et al. Distinct prognosis of idiopathic nonspecific interstitial pneumonia (NSIP) fulfilling criteria for undifferentiated connective tissue disease (UCTD). Respir Med 2010; 104: 1527-1534.

51 Kinder BW, Shariat C, Collard HR, et al. Undifferentiated connective tissue disease-associated interstitial lung disease: changes in lung function. Lung 2010; 188: 143-149. 